\title{
Investigation of Confined Turbulent Jet Flames Using kHz-Rate Diagnostics
}

\author{
Zhiyao Yin*, Isaac Boxx ${ }^{\dagger}$, Michael Stöhr ${ }^{\ddagger}$, Oliver Lammel ${ }^{\S}$ and Wolfgang Meier ${ }^{\Uparrow}$ \\ German Aerospace Center (DLR), Stuttgart, Germany
}

\begin{abstract}
An atmospheric, single-jet combustor with rectangular confinement was previously developed based on the FLOX ${ }^{\circledR}$ concept $^{\mathrm{a}}$. In this study, premixed, preheated $\mathrm{H}_{2}$-air and $\mathrm{CH}_{4}$-air turbulent jet flames were stabilized in this combustor. Self-sustained jet oscillation was observed in both non-reacting and reacting cases. A 3-D and periodic jet flapping is identified via proper orthogonal decomposition (POD) of various 2-D views of the flow field, measured by particle imaging velocimetry (PIV). The frequency of the oscillations and couplings between different Eigenmodes are determined based on results from PIV at $5 \mathrm{kHz}$ repetition rate. The influence of jet flapping on combustion stability is examined in detail using simultaneous PIV/OH chemiluminescence imaging and PIV/planar laserinduced fluorescence of $\mathrm{OH}$ radicals (OH PLIF) at $5 \mathrm{kHz}$ repetition rate. The up/down and expand/contract motions of the lateral recirculation zone (LRZ) due to jet flapping is found to modify the flame shape and flame lift-off height. Such variations are traced to the transformation of low-speed regions in the junction of the back flow and the jet. These regions contain counter flows and vortices with relatively long flow residence time and can therefore enhance the mixing between recirculated hot burned gas and fresh fuel-air mixtures. In some cases, the jet flapping is also found to cause temporary local extinction of the flame, due to reduced entrainment of burned gas. However, the flame is able to recover as the jet flaps away from combustor chamber and reopens the back flow channel.
\end{abstract}

\section{Introduction}

Laminar and turbulent jets under confinement or impingement geometries are known to be prone to selfsustained oscillations. ${ }^{1-3}$ Considerable studies have been dedicated to the characterization of such phenomena due to their presence in a broad range of industries: wind instrument, ${ }^{4}$ ramjet combustor, ${ }^{5}$ fluidic oscillator, ${ }^{6}$ to name a few. The common understanding is that, the oscillations are triggered by obstructed shear layers, and are sustained by a feedback loop between initial disturbances and the impinging points. Such feedback loop is most commonly hydrodynamic through the back flow (e.g., recirculation), as found in jet experiencing sudden expansion ${ }^{7,8}$ and jet issuing into a cavity. ${ }^{3,9,10}$ It could also be of acoustic nature, which may coexist with the hydrodyamic mechanism. ${ }^{1}$ Through parametric studies, oscillations occurred in confined jets have been found to scale with jet velocity and impingement length (e.g., nozzle diameter and confinement dimensions). ${ }^{3,10}$ They often possess a Strouhal number (St) in the range of St 0.001-0.01, much smaller than the instabilities generated within shear layers (St 1$),{ }^{7}$ suggesting the involvement of the jet as a whole.

Advances in particle imaging velocimetry (PIV) in the past decade have allowed topological analysis of jet oscillations in two- and even three-dimensions. In the case of flapping planar jets under rectangular confinement, ${ }^{3,8,11,12}$ a recirculation zone can be found on each side of the jet. As the jet flaps with respect to the plane of symmetry, the two recirculation zones move conversely up or down. At the same time, cross-flow between the nozzle and confinement (e.g., chamber walls) oscillates in response, completing the feedback loop. Through proper orthogonal decomposition (POD) of the 2 -D velocity field, ${ }^{12}$ the spatial mode

\footnotetext{
*Postdoc, Institute of Combustion Technology, Stuttgart, Germany. AIAA Associate Member

†Staff Scientist, Institute of Combustion Technology, Stuttgart, Germany. AIAA Senior Member

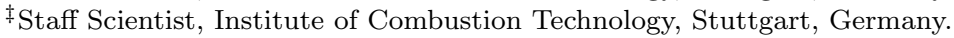

$\S$ Staff Scientist, Institute of Combustion Technology, Stuttgart, Germany.

『 Head of Laser Diagnostics Division, Institute of Combustion Technology, Stuttgart, Germany. AIAA Member

${ }^{\mathrm{a}} \mathrm{FLOX}^{\circledR}$ is a registered trademark of WS Wärmeprozesstechnik GmbH, Renningen, Germany
} 
responsible for the jet flapping has been identified to contain large structures distributed pseudo-periodically alongside the jet. Such observation was consistent with previous analysis based on hot wire measurements. ${ }^{2}$ In the case of an axisymmetric round jet expanding into a concentric cylindrical chamber, ${ }^{7,13,14}$ jet precessing (defined as rotation of the entire jet with respect to the nozzle axis) has been found to induce a swirling flow in the confinement. With tomographic PIV, ${ }^{15}$ it has been shown that asymmetric entrainment induced by a constantly reattaching jet is the cause for the azimuthal pressure gradient, which leads to jet precession. Such dynamic oscillations in confined jets are known to enhance large scale mixing of the jet while also sustaining high turbulent intensity in the far field. ${ }^{8}$ In reacting flows, jet precession has been found to increase flame volume without increasing flame length. ${ }^{16}$ The reduced global flame strain results in an increase of soot production and hence enhanced radiative heat transfer, eventually leading to a decrease in flame temperature and NOx production.

A similar type of jet flapping has been reported in our previous work, ${ }^{17}$ in a confined, single-nozzle gas turbine model combustor, designed ${ }^{18}$ based on the FLOX ${ }^{\circledR}$ concept. FLOX ${ }^{\circledR}$ combustion, also termed as flameless, ${ }^{19}$ volume or mild combustion, ${ }^{20}$ is regarded as a viable alternative to swirl-stabilized flames due to its highly resistance to thermoacoustics and flame flashback, ${ }^{21}$ while also attaining high fuel flexibility. ${ }^{22}$ FLOX ${ }^{\circledR}$ combustors generally consist of circularly arranged nozzles discharging high momentum jets of reactants into a combustion chamber, consequently generating strong recirculation of the flow and intense mixing of reactants and products. In recent years at our institute, significant effort has been devoted to evaluating combustion performance and understanding flame stabilization mechanisms in various FLOX $^{\circledR}$ combustors, both experimentally ${ }^{18,22}$ and numerically. ${ }^{23,24}$ This work focuses on characterizing turbulent jet oscillations and their influences on combustion stability in the single-nozzle combustor. One major emphasis is to time-resolve the jet flapping motion and flame responses using kHz-rate, simultaneous PIV and $\mathrm{OH}$ chemiluminescence imaging or planar laser-induced fluorescence of $\mathrm{OH}$ radical (OH PLIF).

\section{Experimental}

\section{A. Single-nozzle jet-stabilized model combustor}

The single-nozzle model combustor shown schematically in Fig. 1 was developed in our previous work. ${ }^{18}$ Preheated and premixed gas mixture was delivered through a straight stainless steel tube $(\mathrm{ID}=10 \mathrm{~mm}$, $\mathrm{L}=400 \mathrm{~mm}$ ) into the combustor chamber. The nozzle had a chamfered tip and rose $20 \mathrm{~mm}$ from the base plate. The chamber consisted of three identical sections. Each was 200-mm tall and featured a rectangular cross section with an inner dimension of $50 \times 40 \mathrm{~mm}$. The jet nozzle was offset from the geometric center of the combustor chamber, creating an asymmetry in the front view (Fig. 1(b)). The off-center positioning of the jet was designed to imitate the situation around a single nozzle in an actual FLOX ${ }^{\circledR}$ combustor, where a ring of nozzles were placed near the chamber wall, leading to the formation of a large inner recirculation zone. $^{22}$ The whole combustor was mounted on a three-axis translation stage and could be positioned with regard to the laser diagnostics setup. The coordinates used in the following discussions are also defined in Fig. 1.

For this study, the combustor was operated with a various air flow at a preheating temperature of $300^{\circ} \mathrm{C}$ and at 1 bar. Non-preheated methane or hydrogen was added into the main air through a static mixer to achieve various equivalence ratios. The average jet exit velocity was maintained at either $120 \mathrm{~m} / \mathrm{s}$ or $150 \mathrm{~m} / \mathrm{s}$.

\section{B. Low-speed diagnostics}

A commercial particle image velocimetry (PIV) system (LaVision Flowmaster) was used to obtain a global view of the velocity fields in different sections of the combustor chamber. A small portion of the main air (non-preheated) was seeded with titanium dioxide $\left(\mathrm{TiO}_{2}\right)$ particles $(\mathrm{d}=1 \mu \mathrm{m})$ and injected into the static mixer. The second harmonic output of a dual-cavity Nd:YAG laser (with pulse energy of $2 \times 120 \mathrm{~mJ}$ ) was used to illuminate the particles (with a pulse separation of $5 \mu \mathrm{s}$ to $11 \mu \mathrm{s}$ ). The laser beam was expanded into a sheet of 200-mm tall and 1-mm thick and was aligned through the center of the jet nozzle ((x-y or y-z plane, see Fig. 1)). Mie scattering signal from the particles was collected into a CCD camera $(1376 \times 1040$ pixels) coupled with a $\mathrm{f}=50 \mathrm{~mm}$ lenses $(\mathrm{f} / 4)$, a narrow bandpass filter $(532 \pm 5 \mathrm{~nm})$ and a mechanical shutter (to suppress flame

emission). Velocity vectors were calculated using a multipass adaptive window cross correlation algorithm with a final window size of $16 \times 16$ pixels (corresponding to a $2.2 \times 2.2 \mathrm{~mm}^{2}$ spatial resolution). Based on the \pm 0.1 pixel uncertainty of the cross correlation peak-finding algorithm, the uncertainty of the velocity 
Figure 1: Combustor geometry:

(a) Combustor chamber

(b) Front view,

(c) Side view, and

(d) Top view of the bottom section

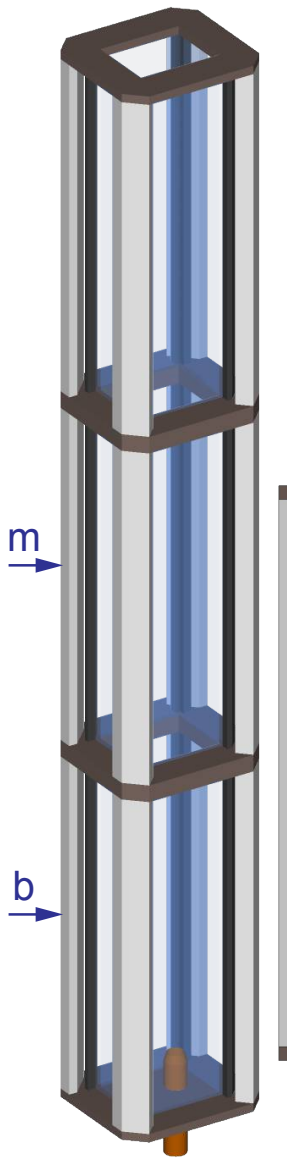

(a)

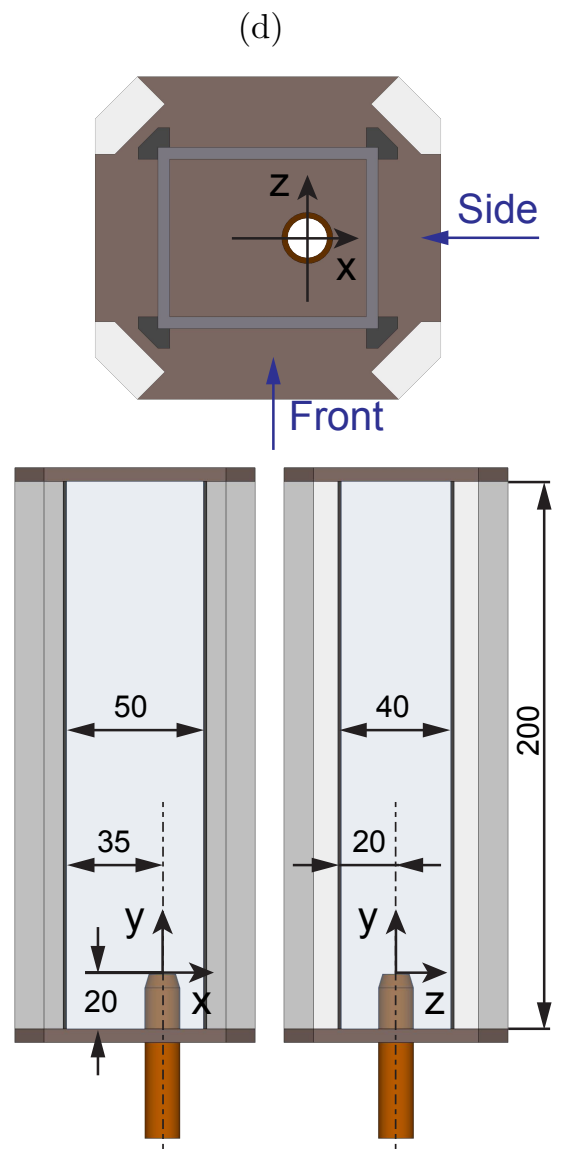

(b)

(c)

measurements was estimated to be $\pm 2.3 \mathrm{~m} / \mathrm{s}$. Separately, $\mathrm{OH}^{*}$ chemiluminescence from the flame was imaged using a combination of a CCD camera, an image relay optics device (IRO), a Cerco UV lens ( $\mathrm{f}=45 \mathrm{~mm}, \mathrm{f} / 1.8$ ) and a bandpass filter centered at $310 \mathrm{~nm}$.

\section{High-speed diagnostics}

As mentioned in Section I, confined jets are known to experience self-excited, self-sustained oscillations, usually leading to a fluctuation of the recirulation zones. Since the recirulation zones play critical roles in flame stabilization in this single-jet combustor, it is important to verify the existence of such instability. For that, $\mathrm{kHz}$-rate laser diagnostics systems at DLR were employed.

Three-component velocity fields were measured using a stereoscopic PIV system at a repetition rate of 5 $\mathrm{kHz}$. The stereo-PIV system was based on a dual-cavity Nd:YAG laser (Edgewave IS-6IIDE, $2.6 \mathrm{~mJ} / \mathrm{pulse}$ at $532 \mathrm{~nm}$ ) and a pair of CMOS cameras (LaVision HSS8) with $\mathrm{f}=200 \mathrm{~mm}$ lenses (f/5.6). Pulse timing separation for the PIV system was set to $10 \mu \mathrm{s}$, resulting in an estimated uncertainty of $\pm 0.2 \mathrm{~m} / \mathrm{s}$. Due to the limitation of laser energy, the laser sheet height was set to about $30 \mathrm{~mm}$. A final field of view (FOV) of approximately $27 \times 25 \mathrm{~mm}^{2}$ was chosen to achieve a higher spatial resolution $\left(0.5 \times 0.5 \mathrm{~mm}^{2}\right.$, with $16 \times 16$ pixels interrogation window) than the low speed measurements described above (the relative size of FOV is shown in Fig.2(c) as the dashed box).

Simultaneously with highspeed PIV, either $\mathrm{OH}^{*}$ chemiluminescence imaging or planar OH Laser-Induced Fluorescence (PLIF) was performed. For $\mathrm{OH}^{*}$ imaging, signal was collected via a Cerco UV lens $(\mathrm{f}=45 \mathrm{~mm}$, f/1.8) coupled with a 310-nm bandpass filter into an intensified CMOS camera (LaVision HSS6 with HSIRO). For OH PLIF, a frequency-doubled dye laser (Sirah Credo) was pumped by the $532 \mathrm{~nm}$ output of an Nd:YAG laser (Edgewave IS-8IIE) to produce $\sim 100 \mu \mathrm{J} /$ pulse at $\sim 282 \mathrm{~nm}$. The laser was tuned to the peak of $\mathrm{Q}_{1}(7)$ line in the $\mathrm{OH} \mathrm{A-X}(1,0)$ band, which is insensitive to temperature in the range of $\mathrm{T}=1200-2000 \mathrm{~K}$ (with only $10 \%$ change in lower state population). The OH PLIF excitation pulse was temporally interlaced between the two PIV pulses. Fluorescence signal was collected with the same camera system used for $\mathrm{OH}$ 
Figure 2: Measured average fields:

(a)\&(b) Streamlines color-coded by velocity magnitude in non-reacting and reacting flows respectively

(c) $\mathrm{OH}$ chemiluminescence

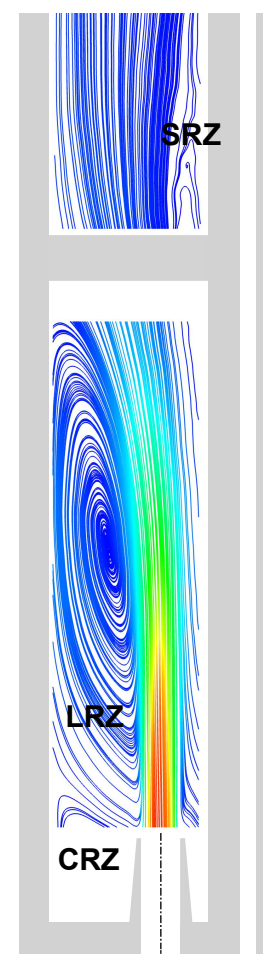

(a)

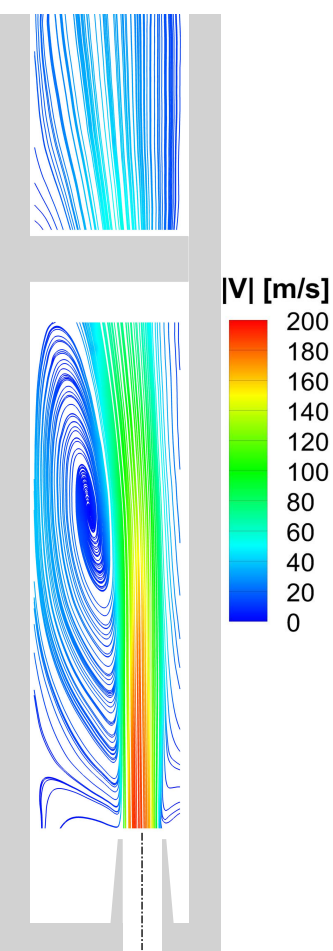

(b)

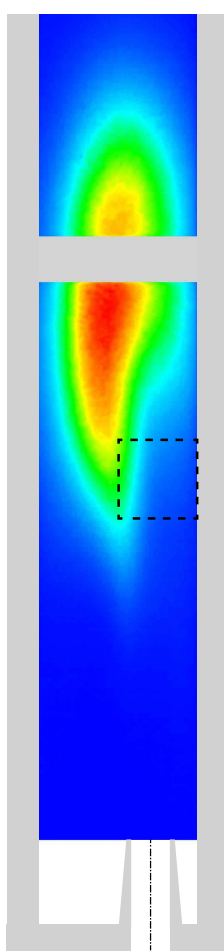

(c)

chemilunimescence imaging, with a FOV of about $40 \times 40 \mathrm{~mm}^{2}$ to overlap with that of the PIV. For both PIV and PLIF, the laser sheets were aligned through the center of the nozzle ( $\mathrm{x}-\mathrm{y}$ plane, see Fig. 1).

\section{Average flow fields and flame shapes}

Figure 2 shows the average flow field and $\mathrm{OH}^{*}$ emission measured at the operating condition from the front view (refer to Fig. 1). Since the flame occupied mainly the bottom section (denoted as ' $b$ ' in Fig.1) and part of the middle section (denoted as ' $c$ ' in Fig.1), the results have been cropped accordingly. Note that the flow field of the bottom and middle sections were obtained from two separate measurements. A good consistency between them is however obviously demonstrated. As reported in our previous work, ${ }^{17,18}$ the most characteristic feature of this combustor is the lateral recirculation zone (LRZ) in the bottom section between the main jet and the chamber wall. This is evident from both the non-reacting (Fig. 2(a)) and reacting (Fig. 2(b)) cases. Two other recirculation zones can also be identified in Fig. 2(a): a corner recirculation zone (CRZ) under the LRZ and a secondary recirculation zone (SRZ) on the opposite side of the jet. The flow fields exhibit similar structures in both non-reacting and reacting cases, except for an overall higher velocity and the disappearance of the SRZ in the reacting case (Fig. 2(b)). With a measured average temperature of $1000-1600 \mathrm{~K}^{18}$ in the reacting case, the LRZ is responsible for entraining large amount of burned gas to mix with the cold jet. Comparing Fig. 2(b) and (c), it is clear that the flame is lifted and stabilized at about midway of the bottom section. This location coincides with the core of the LRZ. In addition, the flame appears strongly asymmetric with respect to the nozzle axis, with a bias towards the LRZ.

Figure 3 shows the influence of equivalence ratio and $\mathrm{H}_{2}$ addition on the structure and lift-off height of the $\mathrm{CH}_{4}$-air flames. For all the cases, air flow rate was adjusted such that the same jet exit velocity of $\mathrm{v}_{j e t}=120$ $\mathrm{m} / \mathrm{s}$ was maintained. It is clear from Fig. 3(a) that equivalence ratio has a small effect on flame lift-off height. However, flame appears to contract with increasing equivalence ratio. On the other hand, Figure 3(b) shows that with increasing $\mathrm{H}_{2}$ mole fraction in the fuel, flame contracts, becomes more symmetric along the nozzle center line, and also moves upstream towards the nozzle.

Flame dynamics can be inferred from frequency analysis of $\mathrm{OH}$ chemiluminescence intensities from a sequence of images. Figure 4 shows power spectral density (PSD) of integrated $\mathrm{OH}$ chemiluminescence (i.e., sum of intensity counts) from a set of 5000 images taken at $5 \mathrm{kHz}$. The integration area covered the entire first section of the combustor (height above jet exit from 0 to $175 \mathrm{~mm}$, see Fig. 3). As shown in Fig. 4(a), 


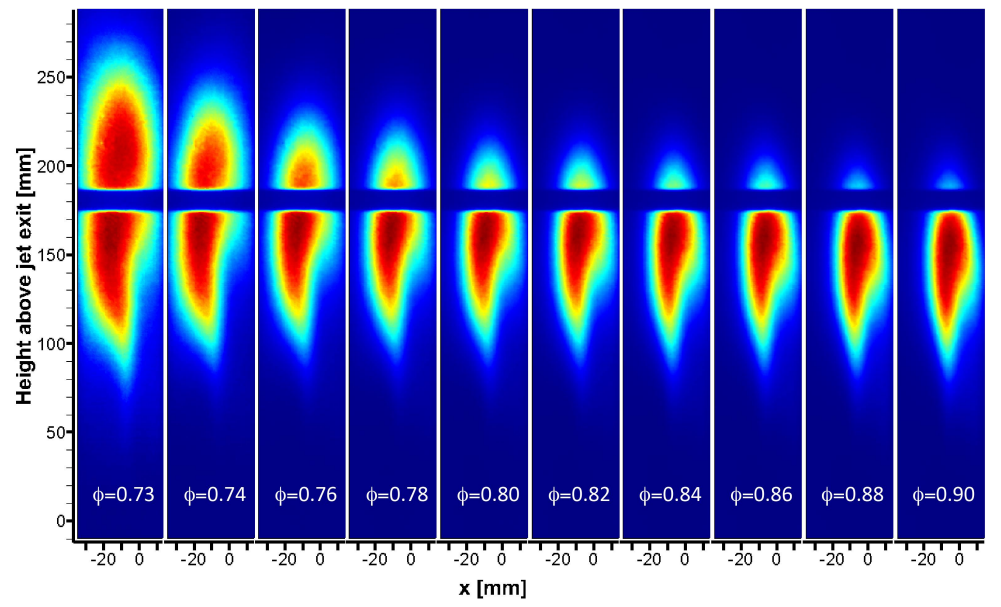

(a)

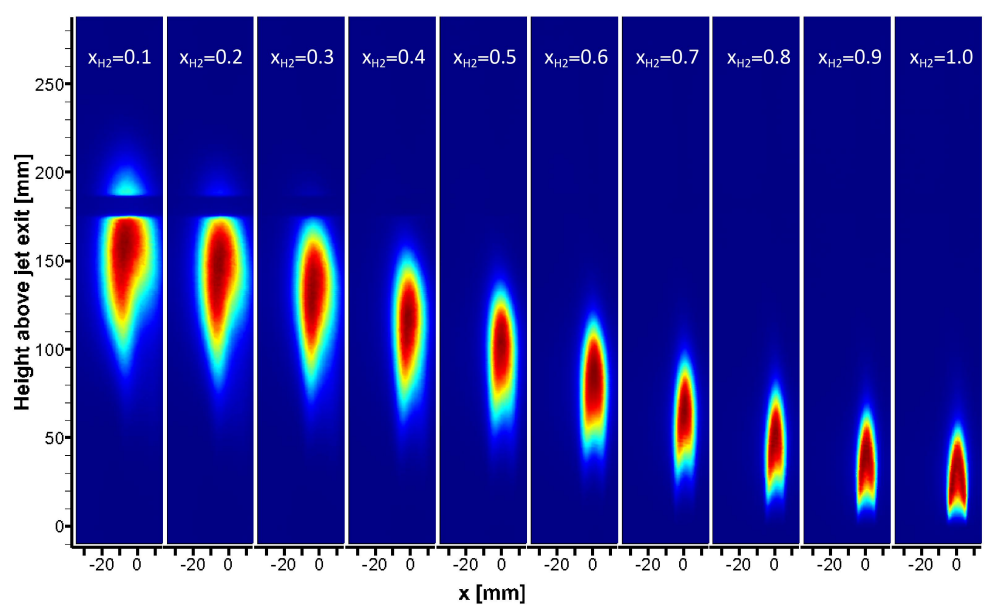

(b)

Figure 3: (a) Average $\mathrm{OH}$ chemiluminescence from $\mathrm{CH}_{4}$-air flames at $\mathrm{T}_{0}=300{ }^{\circ} \mathrm{C}$ and $\mathrm{v}_{\text {jet }}=120 \mathrm{~m} / \mathrm{s}$ with various equivalence ratio. (b) Average $\mathrm{OH}$ chemiluminescence from $\mathrm{CH}_{4}$-air flames at $\phi=0.80, \mathrm{~T}_{0}=300^{\circ} \mathrm{C}$ and $\mathrm{v}_{\text {jet }}=120 \mathrm{~m} / \mathrm{s}$ with different amount of $\mathrm{H}_{2}$ addition. 


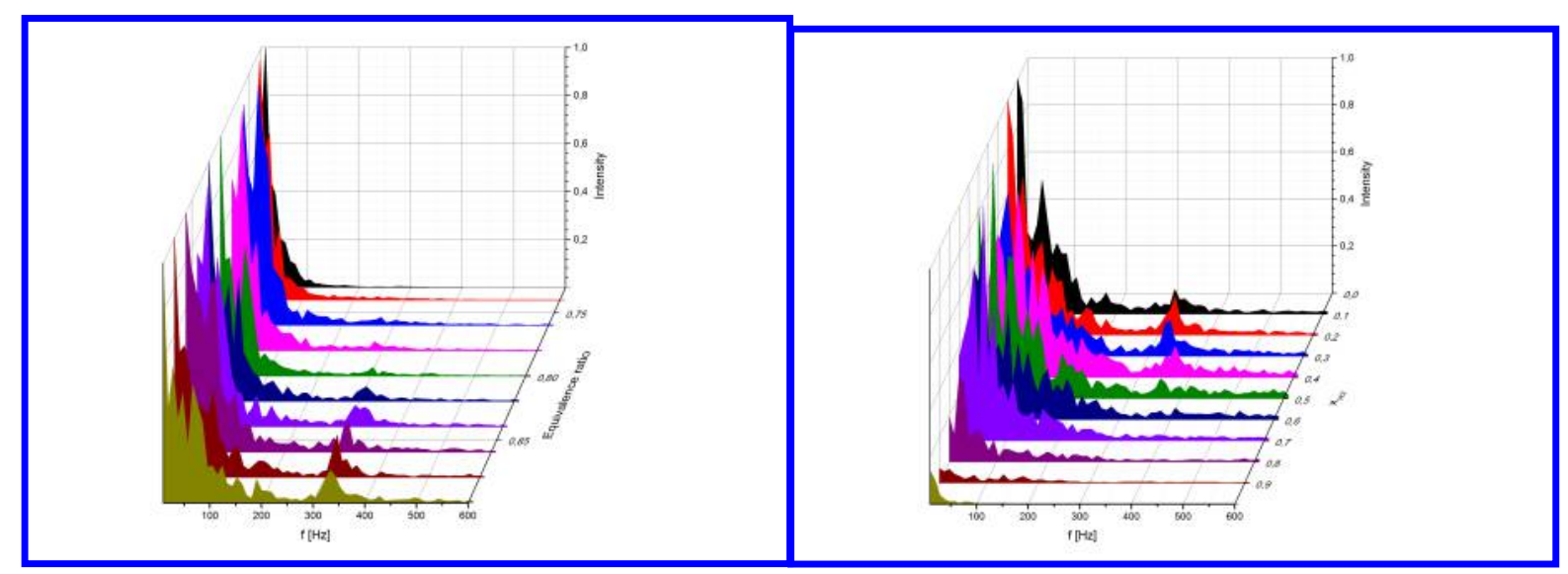

(a)

(b)

Figure 4: (a) PSD of integrated $\mathrm{OH}$ chemiluminescence from a $\mathrm{CH}_{4}$-air flame at $\mathrm{T}_{0}=300{ }^{\circ} \mathrm{C}$ and $\mathrm{v}_{\text {jet }}=120$ $\mathrm{m} / \mathrm{s}$. (b) PSD of integrated $\mathrm{OH}$ chemiluminescence from $\mathrm{CH}_{4} / \mathrm{H}_{2}$-air flames at $\phi=0.80, \mathrm{~T}_{0}=300{ }^{\circ} \mathrm{C}$ and $\mathrm{v}_{\text {jet }}=120 \mathrm{~m} / \mathrm{s}$

a strong fluctuation with a low frequency $(<100 \mathrm{~Hz})$ is visible in all the equivalence ratio cases shown in Fig. 3(a). At $\phi$ larger than 0.80 , an additional high frequency peak $(>300 \mathrm{~Hz})$ starts to appear. From Fig. 4(b), with increasing $\mathrm{H}_{2}$ addition, the instabilities seem to diminish first at $\mathrm{f}>300 \mathrm{~Hz}$ and then at $\mathrm{f}<100$ Hz. It was observed previously in this combustor that the flow field appeared nearly identical for both $\mathrm{CH}_{4}$-air and $\mathrm{H}_{2}$-air flames at the same jet exit velocity. ${ }^{18}$ Therefore the change in flame dynamics shown in Fig. 4 is unlikely a result of change in the flow field. In the case of $\mathrm{H}_{2}$ addition, it is possible that with increasing amount of $\mathrm{H}_{2}$, the flame becomes less susceptible to fluctuations in the flow field.

The following sections focus on one specific operating condition, with a methane-air mixture at $\phi=0.71$,a preheating temperature of $300^{\circ} \mathrm{C}$, and a jet exit velocity at $150 \mathrm{~m} / \mathrm{s}$.

\section{Self-sustained flow field oscillations}

\section{A. Proper orthogonal decomposition (POD) of the flow fields}

As mentioned in Section I, through proper orthogonal decomposition (POD) of the velocity fields, the oscillations of confined planar jets have been associated with pseudo-periodic structures distributed alongside the jet axis. ${ }^{2}, 12$ In this work, a snap-shot method of POD ${ }^{25}$ was first applied to sets of 1000 flow-field measurements carried out with the low-speed PIV system. For the measurements of the side view, the combustor was rotated by $90^{\circ}$, such that the laser sheet crossed the y-z plane (see Fig. 1).

The first two spatial Eigenmodes of the flow field for both the front and side views of the combustor are shown as color-coded streamlines in Fig. 5. The contributions of the Eigenmodes to the total energy in the flow field are plotted in Fig. 6. It is clear that the first mode is also the most dominant mode (referred to in this work as the 'lateral mode'), with alternating planar structures on each side of the average jet trail (see Fig. 2) with opposite vector directions (indicative from the recirculation). These structures are consistent with those observed in a confined planar jet, ${ }^{12}$ which were linked to periodic jet flapping. The second mode appears to be pointing to a completely different motion: with a single peak in the velocity magnitude on the jet trail, suggesting a vertical movement of the jet body (and is termed the 'axial mode'). The third and fourth modes (not shown in Fig.5) are the mode pairs to the lateral and axial modes. They have similar spatial structures as their stronger correspondents, but contribute much less to the total energy.

\section{B. Low-order modeling of phase-dependent flow fields}

The nature of the self-sustained oscillations identified by the POD Eigenmodes can be visualized by modeling phase-dependent flow fields. This can be done by utilizing the average flow field and the spatial Eigenmode in interest, as demonstrated in. ${ }^{26}$ In the case under study here, since the lateral mode is by far the strongest, it is reasonable to approximate the flapping motion by considering the lateral mode alone. 
The phase dependent flow field $\vec{u}(\psi)$ modeled by considering a single Eigenmode can be written as:

$$
\vec{u}(\psi) \approx\langle\vec{u}\rangle+\sqrt{\lambda_{i}} \cdot \sin \psi \cdot \vec{e}_{i},
$$

where $\langle\vec{u}\rangle$ is the average flow field, $\psi$ is the phase angle, $\lambda_{i}$ is the Eigenvalue of the Eigenmod $\vec{e}_{i}$ and can be expressed by the temporal coefficient $a_{j i}$ of Mode $i$ from each snap shot $j$ :

$$
\lambda_{i}=\frac{1}{N} \cdot \sum_{j=1}^{N} a_{j i}^{2},
$$

where $N$ is total number of snap shots used for POD.

Figure 7 shows the phase-dependent non-reacting flow fields, modeled based on the front lateral mode shown in Fig. 2(a), by using Eq. 1. Note that, per definition, the flow field at $\psi=0^{\circ}$ and $180^{\circ}$ recovers to the average flow field $\langle\vec{u}\rangle$. In the first half cycle, the jet flaps leftwards, pushing the LRZ downwards. In the meantime, the SRZ, which is barely visible in the averaged flow field $\left(\psi=0^{\circ}\right)$, increases in size and reaches its maximum at $\psi=90^{\circ}$. Then the jet flaps rightwards, back to the original position. This rightward flapping continues into the second half of cycle, until the jet is almost "straightened up" at $\psi=270^{\circ}$, accompanied by the upward motion of the LRZ and the disappearance of the SRZ. In a similar fashion, the oscillation in the side view can also be modeled, as shown in Fig. 7. From the side view, the jet can also be seen flapping towards each wall, resulting in upward/downward motion of the recirulation zones on each side of the jet. This is more resemblance to the flapping motions observed in the symmetric planar jets. ${ }^{12}$ These results suggest that the flapping motion could be observable in any cut-through plane with regard to the nozzle axis (y-axis).

The same modeling method was also conducted by employing the axial modes for both the front and side views. Although this mode is relatively weak and could be coupled with other modes, the aim is to reveal the flow pattern controlled by the axial mode. The results are shown in Fig. 8. The middle section exhibited little variance at different phases and was therefore not included in the plot. It seems the axial mode is associated with a fluctuation causing the far field of the jet to go through an increasing/decreasing cycle. This is evident from the expansion/contraction of the green region in the streamlines in Fig. 8. The core area of the jet (red), however, does not seem to be affected, suggesting that this axial fluctuation is not a pulsating motion along the nozzle axis. Considering the concurring jet flapping in the combustor chamber, this axial fluctuation could be explained as the manifestation of the jet moving in and out of the observation plane (i.e., the cut-through plane of the laser sheet). In this sense, the lateral mode in the side view (Fig. 7) can be considered as essentially inducing the axial mode in the front view (Fig. 8). Therefore, the coupling between the in-plane and out-of-plane motion can be derived by comparing the lateral and axial modes in the same view, as detailed in the following section.

\section{Mode coupling}

As shown in, ${ }^{27}$ two Eigenmodes (representing the real and complex parts) are needed to describe a traveling wave. The flapping motion in a confined planar jet (a 2-D flow) was also shown to be controlled by two Eigenmodes with similar energy contributions but a phase shift of their temporal coefficients in the frequency domain. ${ }^{12}$ As already mentioned in Section A, the third and fourth Eigenmodes were found to have similar spatial structures as the lateral and axial modes. To shed light on the complex asymmetric and three dimensional jet motion in the combustor, the temporal behaviors of various spatial modes and their interactions need to examined. This is done by time-resolving the flapping motion using the $\mathrm{kHz}$ diagnostic system described in Section II.

Figure 9(a) shows the lateral modes obtained from decomposing a set of 3750 shots of velocity fields obtained at $5 \mathrm{kHz}$, plotted on the same color scale as 5 . Even though the FOV is much smaller, the spatial Eigenmodes and their energy contributions correspond well to those taken with the low-speed system (Fig. 5 and Fig. 6). The companion lateral mode, denoted by a prime sign, exhibit a more convoluted flow field with much lower velocity magnitude. The correlation between the two modes is much clearer from their temporal coefficients ( $a$ in Eq.2). As shown in Fig. 9(b), the weaker mode $\left(a_{\text {Lateral }}^{\prime}\right)$ lags behind the primary mode ( $\left.a_{\text {Lateral }}\right)$ by roughly $1 / 4$ of a cycle, as expected between a mode pair. The similarity between the mode pair can also be found in the power spectral density (PSD) of the temporal coefficients, plotted in Fig. 9(c)

the strongest peak can be found at the same frequency. The same observations were made regarding the two axial modes, the results therefore are omitted here. 
In addition to the coupling between the mode pairs, coupling between the lateral and the axial modes was also identified. From their temporal coefficients plotted in Fig.8 10(a) ( $a_{\text {axial }}$ smoothed with a $\delta t=0.01 \mathrm{~s}$ window to remove high frequency noise), the axial mode appears to have doubled the time period as the lateral mode. This is confirmed from PSD of their temporal coefficients given in Fig.8 10(b). The axial mode peaks at $19.9 \mathrm{~Hz}$, exactly half of the strongest peak found in the lateral mode. Furthermore, the peaks and valleys in the axial mode (correspond to phase angle of $90^{\circ}$ and $270^{\circ}$ seem to always coincide with the peaks in the lateral mode. A Lissajous plot of their temporal coefficients shows that the temporal coefficients do not scatter randomly in the phase portrait but rather appear to be bounded within a semi-parabolic trajectory. It seems when the jet swings one cycle in and out of the measurement plane (axial mode), it flaps left and right (in-plane) two complete cycles (lateral mode).

These results can be used to reconstruct the 3-D jet flapping motion inside the combustor, as illustrated schematically in Fig. 10(d). A likely projection of the jet flapping motion from the top view is represented by the red dashed line with regard to the jet nozzle. Based on Fig. 10(a), the corresponding phase angles at various jet locations are also marked out with respect to the front and the side views of the combustor chamber (refer to Fig. 1(d)). As the jet moves along the red dashed line, it completes two cycles of jet flapping from the perspective of the front view. While from the side view, it only flaps one cycle.

It should be emphasized that the simple jet flapping track illustrated in Fig. 10(d) is only an idealized case. It is presented to demonstrate the 3-D nature of the jet oscillation inside the combustor chamber. As is obvious from the pseudo-sinusoidal behavior of the temporal coefficients in Fig. 10(b) and the rather noisy phase portrait in Fig. 10(d), the exact location of the jet at different flapping cycles can follow various tracks, which is also not necessarily symmetric to the nozzle axis within the same cycle.

\section{Influence of reaction on the flow field instability}

Since the jet oscillation is associated with density (pressure) imbalances around the jet, combustion reactions may exert influences on the flow field and its instability. In the reacting case, small differences can be observed in the average flow field shown in Fig. 2 (see Section B). POD Eigenmodes exhibit identical spatial distributions for both cases, with the same ordering of energy contributions. In the reacting case, the SRZ appears in phase modeling of the flow field using the lateral mode and increases in size until phase $90^{\circ}$. Similar mode coupling between the lateral and axial modes also exists in the reacting case, but appears mirrored (i.e., with a $180^{\circ}$ phase shift) from the non-reacting case, as shown in Fig. 11. Most notable difference in the reacting case is the appearance of multiple peaks in the PSD of the temporal coefficients, as plotted in Fig. 11(b). These peaks are also much weaker than in the non-reacting case.

It is clear that in the reacting flow, the jet oscillation becomes less coherent and more chaotic. This kind of coexistence of various frequencies has been previously documented. ${ }^{1}$ In the current study, the multifrequency feature could stem from a feedback between the jet oscillation and combustion reactions (and heat release). The former one may alter flame structure and flame lift-off height, which may then trigger a change in local heat release, leading to a shift of the density balance in the chamber and subsequently affecting the jet flapping. After a close look at the time-resolved experimental results, these different peaks can all be related to the same general motion of jet flapping (as modeled in Fig. 7), but at different pace (cycle).

\section{Flow oscillation induced combustion instabilities}

This section focuses on the influences of the jet flapping on combustion stability. The results from $5 \mathrm{kHz}$ measurements were grouped and averaged based on phase angles extracted from the temporal coefficients of the lateral mode of the flow field, such as the one shown in Fig. 9(b). A zero-crossing detection algorithm was used to assign $0^{\circ}$ or $180^{\circ}$ to corresponding frames, assuming a sinusoidal behavior of the temporal coefficients (to match the phase angles used in Section B). Then the phase angles were extrapolated accordingly for frames in between of zero crossings. A low pass filter was introduced in the zero-crossing algorithm to discard frames that fall into fluctuations larger than $100 \mathrm{~Hz}$. An entire cycle was then divided into eight groups, each spanning a range of $45^{\circ}$. This information was then used for phase averaging.

\section{A. Influence on flame shape}

From measurements of simultaneous PIV and $\mathrm{OH}$ chemiluminescence imaging at $5 \mathrm{kHz}$. The $\mathrm{OH}^{*}$ images were phase averaged using the method described above, based on the temporal coefficients obtained from 
POD of simultaneously measured velocity field.

Figure 12(a) shows the phase-averaged $\mathrm{OH}^{*}$ emission at the four phase angles corresponding to the jet flapping shown in Fig. 7. Only part of the bottom section of the combustor was imaged to focus on the root of the flame. The contour line at signal counts of 20 was also drawn (black line) to qualitatively illustrate the boundary of the flame. As expected, the flame shape at $0^{\circ}$ and $180^{\circ}$ resembles that in the average $\mathrm{OH}$ chemiluminescence shown in Fig. 2. As the jet flaps leftwards (i.e., the LRZ) to reach phase $90^{\circ}$, the flame extends along the right wall further upstream, likely due to the increase of SRZ shown in Fig. 7, which may bring more burned gas upstream along the right wall. Also, the flame lift-off height reduces slightly and becomes more attached to the left wall. This coincides with the left- and downward motion of the LRZ, shown in Fig. 7. On the contrary, during the second half of the cycle, as the jet straightens up, the flame becomes more detached from the left wall and the flame lift-off height also increases slightly. Again, this corresponds well to the right- and upward motion of the LRZ. Further more, due to the disappearance of the SRZ, the flame near the right wall recedes downstream.

In fact, the flame root is more dynamic than the phase averaged images give credit to. This is demonstrated in Fig. 12(b), in which representative instantaneous $\mathrm{OH}$ chemiluminescence images from each phase group are shown. The flame shape varies notably from their corresponding phase average. This is likely a result of the multi-frequency jet flapping in the reacting flow discussed in Section D. The jet flapping intensiveness (pace and tilt degree) can vary from cycle to cycle. Therefore, its influence on the flame structure can vary even in the same phase group. Since the phase averaging lumps all lower frequencies together, it likely subdued the extreme variations in certain cases. A phase averaging inclusive of only a small frequency range would result in more pronounced variances from phase to phase. Nevertheless, Fig. 12 demonstrates that the movement of the LRZ occurred due to jet flapping modifies the flame structure quite notably.

\section{B. Influence on reaction zones}

Flow-flame interactions were further examined at a finer scale with simultaneous PIV and OH PLIF measurements at $5 \mathrm{kHz}$. Figure 13(a) overlaps the phase averaged velocity field (mono-color streamlines) with $\mathrm{OH}$ distribution (color maps). The $\mathrm{OH}$ signal was not corrected for laser absorption or fluorescence trapping. However, these effects are considered rather minor in this lean and low-temperature flame (with a measured average temperature of up to 1700 to $1800 \mathrm{~K}^{18}$ ). The plot at each phase is stitched together from separate measurements, although good convergence can be observed at the boundaries of the FOVs (at $\mathrm{x}=-10 \mathrm{~mm})$.

From top to bottom of Fig. 13(a), the jet is seen to swing left and right, accompanied by a movement of the LRZ and appearance/disappearance of SRZ, same as observed in the low-order modeling of the flow field shown in Fig. 7. Such resemblance also lends support to the legitimacy of the low-order modeling in Section B. This is accompanied by the shifting of the $\mathrm{OH}$ boundary (zero intensity counts of $\mathrm{OH}$ signal). Comparing to the phases of $0^{\circ}$ and $180^{\circ}$, OH signal concentrates more towards the left wall at $90^{\circ}$, while more near the boundary at $270^{\circ}$. Near the right wall, $\mathrm{OH}$ intensity increases as the SRZ appears, matching the trend shown in $\mathrm{OH}$ chemiluminescence seen in Fig. 12.

Fig. 13(b) presents a different perspective of the same phenomena. The velocity magnitude is used to illustrate the low velocity region (dark blue) in the field. The motion of the LRZ is clearly marked by the expansion $\left(90^{\circ}\right)$ and shrinkage $\left(270^{\circ}\right)$ of the low velocity region in the given FOV, together with a reduction $\left(90^{\circ}\right)$ and increase $\left(270^{\circ}\right)$ of the back flow near the left wall. The boundary of the $\mathrm{OH}$ distribution shown in Fig. 13(a) is outlined qualitatively (with a contour line of counts=200) on top of the velocity field. It is clear that the flame boundary transforms with the low velocity region. This indicates that the recirculation zones (LRZ and SRZ) not only entrain hot burned gas upstream, but also create low velocity regions that allow relatively long residence time for better mixing of the burned gas and the fresh air-fuel mixtures, and hence sustain the flame. The observation in Fig. 12 can then be explained. As the jet oscillates, it influences the size and location of the LRZ and therefore destabilizes the flame, causing the flame lift-off height to shift downwards $\left(90^{\circ}\right)$ and upwards $\left(270^{\circ}\right)$. Note that, this also explains the asymmetry seen in the flame shape in general. The bias mentioned in describing Fig. 2 is a direct result of the low-speed regions in the LRZ, hence the spatial coincidence of the flame root and the core of LRZ.

A detailed view at the influence of jet flapping on the $\mathrm{OH}$ distribution is provided in Fig. 14. Instantaneous velocity fields and $\mathrm{OH}$ signal distributions, selected from two extreme phase groups $\left(90^{\circ}\right.$ and $\left.270^{\circ}\right)$, are overlapped to illustrate the flow-flame interactions in the LRZ and SRZ. Note that Fig. 14(a) and (b) are from two different set of measurements. The regions containing low intensity $\mathrm{OH}$ (green-blue) are mostly accompanied by back flow (refer to vector directions), suggesting that they are mainly recirculated burned 
gas. On the other hand, the regions of distinctively high $\mathrm{OH}$ signal (red) likely come from superequalibrium $\mathrm{OH}$ and can be treated as a qualitatively marker of the reaction zones. As can be seen, the reaction zones always distribute along the junctions of the high-velocity jet and the back flow, where opposing flow or vortices can be identified. These regions also shift along side the jet flapping motion, as evident from Fig. 14(a) (from top to bottom row). Additionally, at phase angle of $90^{\circ}$, the reaction zones appear wider spread, likely a result of longer flow residence time (i.e., better mixing of burned and unburned gas) in the LRZ, as shown in Fig. 13. The opposite can be seen in the case at phase angle of $270^{\circ}$, due to the increase of the back flow and thinning of low velocity region. It should be pointed out that the region of high $\mathrm{OH}$ intensity (red) do not correlate directly to the actual thickness of the reaction zone.

The influence of the SRZ is shown in Fig. 14(b). As the SRZ forms when the jet flaps leftwards $\left(90^{\circ}\right)$, an additional pathway for burned gas entrainment is created opposite to the main flame stabilization region (i.e., LRZ). Regions with low flow velocity give rise to increased reactivity, as is evident from the spots of high intensity $\mathrm{OH}$ signal. Such pathway is completely closed when the jet flaps to the upright position $\left(270^{\circ}\right)$.

\section{Local flame extinction}

From the results shown above, it is tentative to draw a conclusion that the leftwards jet motion may benefit flame stabilization by creating large regions with low flow velocity. However, it should be noticed that the leftward jet flapping essentially reduces the entrainment of the burned gas upstream. One can imagine some extreme scenarios where the impingement of the jet on the combustor wall would cause blockage of the backflow. A such scenario is the SRZ. Flame distinguishes in the region when the jet flaps rightwards, as shown in Fig. 14(b). For the LRZ, it was also observed that in certain cases the jet could temporarily reduce the entrainment of the burned gas as it flaps leftwards, resulting in local flame extinction. An example is provided in Fig. 15. A sequence of instantaneous velocity fields and $\mathrm{OH}$ distributions is shown with an arbitrary time zero and their corresponding phase angles labeled above each frame (all from the group of phase $90^{\circ}$ ). The velocity fields were low-order reconstructions by using the first 5 POD modes (total energy contribution about $25 \%$ ). This is done analogous to Eq. 1, but with the actual temporal coefficients replacing the $\sqrt{\lambda_{i}} \cdot \sin \psi$ term and additional Eigenmodes $\vec{e}_{i}(i=1: 4)$. This is to smooth out small flow structures and focus only on the jet flapping. As can be seen, when the jet moves leftwards, the low speed region retreats downstream, together with a slow down of the back flow. The partial blockage of the back flow causes the continuous flame to first break into two parts, and results eventually in significant fragmentation of the flame in the FOV.

However, the flame is able to recover quickly in the FOV following the rightward swinging of the jet, as depicted by a later sequence of frames in Fig. 16. This sequence happens approximately $3 \mathrm{~ms}$ after those in Fig. 15 and belongs to the next phase group of $135^{\circ}$. From Fig. 16, the reentry of burned gas into the FOV enables a lot of high reactive regions (red). This could be attributed to rather abundant unburned gas retained in this region after the temporary local extinctions. Over time, these red regions dissipate until a continuous flame is reestablished in the FOV. Such unstable behavior could account for the sometimes volatile changes in the flame root, as described in Fig. 12(b).

\section{Conclusions}

This paper presented experimental studies of confined, premixed, preheated turbulent flames, generated in a single-nozzle jet-stabilized gas turbine model combustor. The focus was placed upon the characterization of self-sustained flow field oscillations in the combustor, and their influence on combustion stability in a methane-air flame at $\phi=0.71$, a preheating temperature of $300^{\circ} \mathrm{C}$, and a jet exit velocity at $150 \mathrm{~m} / \mathrm{s}$.

Various views of the flow field inside the combustor measured with both low-speed and $\mathrm{kHz}$ particle imaging velocimetry (PIV) displayed a pronounced recirculation zone on one side of the jet, due to the off-center placement of the nozzle. Analyses based on proper orthogonal decomposition (POD) of the flow fields associated four strongest Eigenmodes to a 3-D jet flapping motion. The jet flapping was also seen accompanied by an up/down, contract/expand motion of the recirculation zones. Combustion reactions have in general small influences on the average flow field and the jet flapping. However, the POD temporal coefficients exhibit multiple peaks in the frequency domain, compared to rather monophonic behavior in the non-reacting case. These different peaks were seen all related to the same general motion of jet flapping, but 
at various paces.

From simultaneous PIV and $\mathrm{OH}$ chemiluminescence imaging at $5 \mathrm{kHz}$, both phase averaged and singleshot $\mathrm{OH}^{*}$ distributions show a notable variance of flame shape and flame lift-off height. During one flapping cycle, the flame changed from extending upstream along the walls, to being lifted-off further. Further examination of such behavior was carried out with simultaneous PIV and planar laser-induced fluorescence of $\mathrm{OH}$ radicals $(\mathrm{OH} \mathrm{PLIF})$. A correlation was found between regions of high $\mathrm{OH}$ intensity and low-speed flow (due to recirculation), the latter was viewed as providing relatively long residence time and beneficial for enhanced mixing of entrained burned gas and fresh gas mixtures. The low-speed regions shifted with the motion of the recirculation zones during the jet flapping cycle, causing the changes in the flame shape and its lift-off height.

From instantaneous $\mathrm{OH}$ distributions selected from the two extreme phase groups $\left(90^{\circ}\right.$ and $\left.270^{\circ}\right)$, the reaction zones (or regions with high reactivity) were seen distributed between the junction of back flow (hot burned gas) and the unburned jet, where vortices and regions of counter flows (sources of low-speed flow) could be identified. The spread of the reaction zones also seemed to expand and contract following similar motions of the recirulation zones, consistent with the phase averaged results. A potential flame-destabilizing pattern was found in a sequence of instantaneous highspeed images of $\mathrm{OH}$ and velocity field. It was seen that jet flapping towards the confinement resulted in a temporary and partial blockage of the entrainment of burned gas into the LRZ, leading to local flame extinction. However, the flame was able to quickly recover, due to the increase of back flow as the jet flaps away from the chamber wall.

\section{Acknowledgments}

Zhiyao Yin acknowledges the financial support within the Helmholtz Postdoc Programme (Grant PD112).

\section{References}

${ }^{1}$ A. Maurel, P. Ern, B. Zielinska, and J. Wesfreid, "Experimental study of self-sustained oscillations in a confined jet," Physical Review E, vol. 54, no. 4, p. 3643, 1996.

${ }^{2} \mathrm{~S}$. V. Gordeyev and F. O. Thomas, "Coherent structure in the turbulent planar jet. part 1. extraction of proper orthogonal decomposition eigenmodes and their self-similarity," Journal of Fluid Mechanics, vol. 414, pp. 145-194, 2000.

$>{ }^{3}$ N. Lawson and M. Davidson, "Self-sustained oscillation of a submerged jet in a thin rectangular cavity," Journal of fluids and structures, vol. 15, no. 1, pp. 59-81, 2001.

${ }^{4}$ M.-P. Verge, B. Fabre, W. Mahu, A. Hirschberg, R. Van Hassel, A. Wijnands, J. De Vries, and C. Hogendoorn, "Jet formation and jet velocity fluctuations in a flue organ pipe," The Journal of the Acoustical Society of America, vol. 95, no. 2, pp. 1119-1132, 1994.

${ }^{5}$ K. H. Yu, A. Trouv, and J. W. Daily, "Low-frequency pressure oscillations in a model ramjet combustor," Journal of Fluid Mechanics, vol. 232, pp. 47-72, 111991.

${ }^{6}$ T. Shakouchi, "A new fluidic oscillator, flowmeter, without control port and feedback loop," Journal of Dynamic Systems, Measurement, and Control, vol. 111, no. 3, pp. 535-539, 1989.

${ }^{7}$ G. Nathan, S. Hill, and R. Luxton, "An axisymmetric 'fluidic' nozzle to generate jet precession," Journal of Fluid Mechanics, vol. 370, pp. 347-380, 1998.

${ }^{8}$ J. Mi, G. Nathan, and R. Luxton, "Mixing characteristics of a flapping jet from a self-exciting nozzle," Flow, turbulence and combustion, vol. 67, no. 1, pp. 1-23, 2001.

${ }^{9}$ R. Schwarze, J. Klostermann, and C. Brücker, "Experimental and numerical investigations of a turbulent round jet into a cavity," International Journal of Heat and Fluid Flow, vol. 29, no. 6, pp. 1688-1698, 2008.

${ }^{10}$ A. Mataoui and R. Schiestel, "Unsteady phenomena of an oscillating turbulent jet flow inside a cavity: Effect of aspect ratio," Journal of Fluids and Structures, vol. 25, no. 1, pp. 60-79, 2009.

${ }^{11}$ B. Kong, M. Olsen, R. Fox, and J. Hill, "Population, characteristics and kinematics of vortices in a confined rectangular jet with a co-flow," Experiments in fluids, vol. 50, no. 6, pp. 1473-1493, 2011.

${ }^{12}$ O. Semeraro, G. Bellani, and F. Lundell, "Analysis of time-resolved piv measurements of a confined turbulent jet using pod and koopman modes," Experiments in fluids, vol. 53, no. 5, pp. 1203-1220, 2012.

13 J. Mi and G. Nathan, "Self-excited jet-precession strouhal number and its influence on downstream mixing field," Journal of fluids and structures, vol. 19, no. 6, pp. 851-862, 2004.

${ }^{14}$ J. Mi, G. Nathan, and C. Wong, "The influence of inlet flow condition on the frequency of self-excited jet precession," Journal of fluids and structures, vol. 22, no. 1, pp. 129-133, 2006.

${ }^{15}$ G. Cafiero, G. Ceglia, S. Discetti, S. Ianiro, T. Astarita, and G. Cardone, "The three-dimensional swirling flow past a sudden expansion," in PIV13; 10th International Symposium on Particle Image Velocimetry, Delft, The Netherlands, July 1-3, 2013, Delft University of Technology, Faculty of Mechanical, Maritime and Materials Engineering, and Faculty of Aerospace Engineering, 2013. 
${ }^{16}$ G. Nathan, J. Mi, Z. Alwahabi, G. Newbold, and D. Nobes, "Impacts of a jet's exit flow pattern on mixing and combustion performance," Progress in Energy and Combustion Science, vol. 32, no. 5 - 6, pp. 496 - 538, 2006.

${ }^{17}$ Z. Yin, P. Nau, I. Boxx, and W. Meier, "Characterization of a single-nozzle FLOX ${ }^{\circledR}$ model combustor using khz laser diagnostics," in Proc. ASME Turbo Expo 2015, Power for Land, Sea and Air, June 15-19, Montreal, Canada, American Society of Mechanical Engineers, 2015.

$\checkmark{ }^{18}$ O. Lammel, M. Stöhr, P. Kutne, C. Dem, W. Meier, and M. Aigner, "Experimental analysis of confined jet flames by laser measurement techniques," Journal of Engineering for Gas Turbines and Power, vol. 134, no. 4, p. 041506, 2012.

${ }^{19} \mathrm{~J}$. Wünning and J. Wünning, "Flameless oxidation to reduce thermal no-formation," Progress in energy and combustion science, vol. 23, no. 1, pp. 81-94, 1997.

${ }^{20}$ A. Cavaliere and M. de Joannon, "Mild combustion," Progress in Energy and Combustion science, vol. 30, no. 4, pp. 329366, 2004.

$\checkmark{ }^{21}$ R. Lückerath, W. Meier, and M. Aigner, "FLOX ${ }^{\circledR}$ combustion at high pressure with different fuel compositions," Journal of Engineering for Gas Turbines and Power, vol. 130, no. 1, p. 011505, 2008.

${ }^{22}$ O. Lammel, H. Schütz, G. Schmitz, R. Lückerath, M. Stöhr, B. Noll, M. Aigner, M. Hase, and W. Krebs, "FLOX ${ }^{\circledR}$ combustion at high power density and high flame temperatures," Journal of Engineering for Gas Turbines and Power, vol. 132, no. 12, p. 121503, 2010.

${ }^{23}$ H. Schütz, R. Lückerath, B. Noll, and M. Aigner, "Complex chemistry simulation of FLOX ${ }^{\circledR}$ : Flameless oxidation combustion," International Journal of Energy for a Clean Environment, vol. 8, no. 3, 2007.

${ }^{24}$ H. Schütz, R. Lückerath, T. Kretschmer, B. Noll, and M. Aigner, "Analysis of the pollutant formation in the FLOX ${ }^{\circledR}$ combustion," Journal of Engineering for Gas Turbines and Power, vol. 130, no. 1, p. 011503, 2008.

${ }^{25}$ G. Berkooz, P. Holmes, and J. L. Lumley, "The proper orthogonal decomposition in the analysis of turbulent flows," Annual review of fluid mechanics, vol. 25, no. 1, pp. 539-575, 1993.

${ }^{26}$ M. Stöhr, R. Sadanandan, and W. Meier, "Phase-resolved characterization of vortex-flame interaction in a turbulent swirl flame," Experiments in Fluids, vol. 51, no. 4, pp. 1153-1167, 2011.

${ }^{27}$ D. Rempfer and H. F. Fasel, "Evolution of three-dimensional coherent structures in a flat-plate boundary layer," Journal of Fluid Mechanics, vol. 260, pp. 351-375, 21994. 
Figure 5: POD spatial modes of the nonreacting flow field:

(a) Front lateral mode

(b) Front axial mode

(c) Side lateral mode

(d) Side axial mode

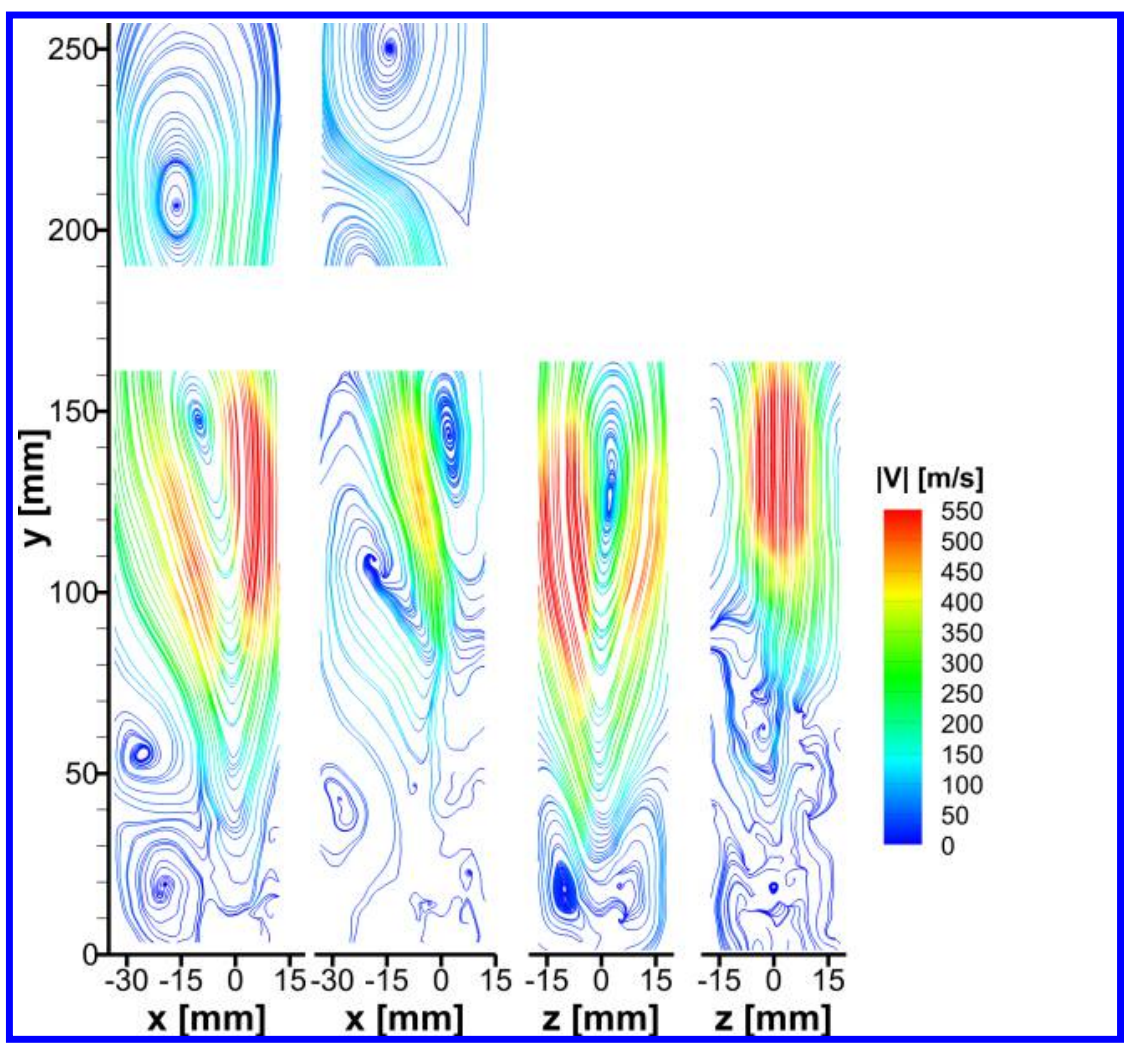

(a) (b) (c)

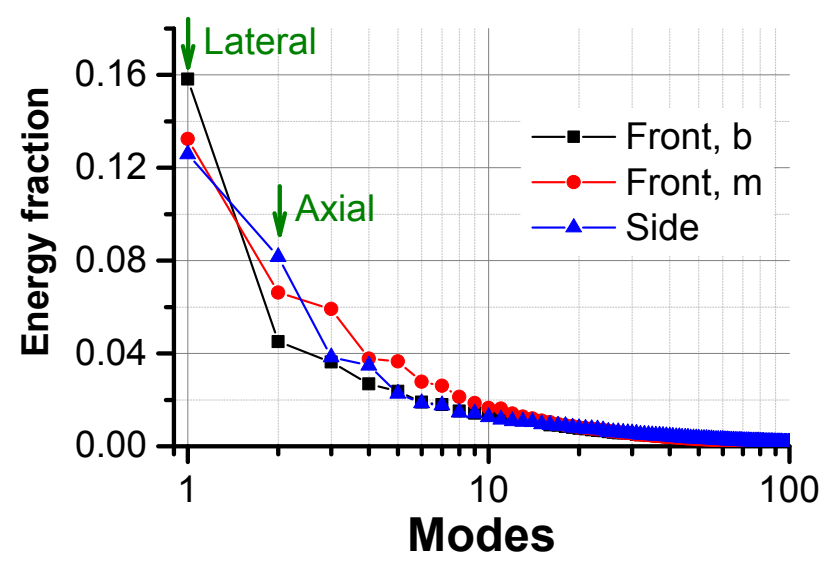

Figure 6: Energy fraction of POD Eigenmodes 


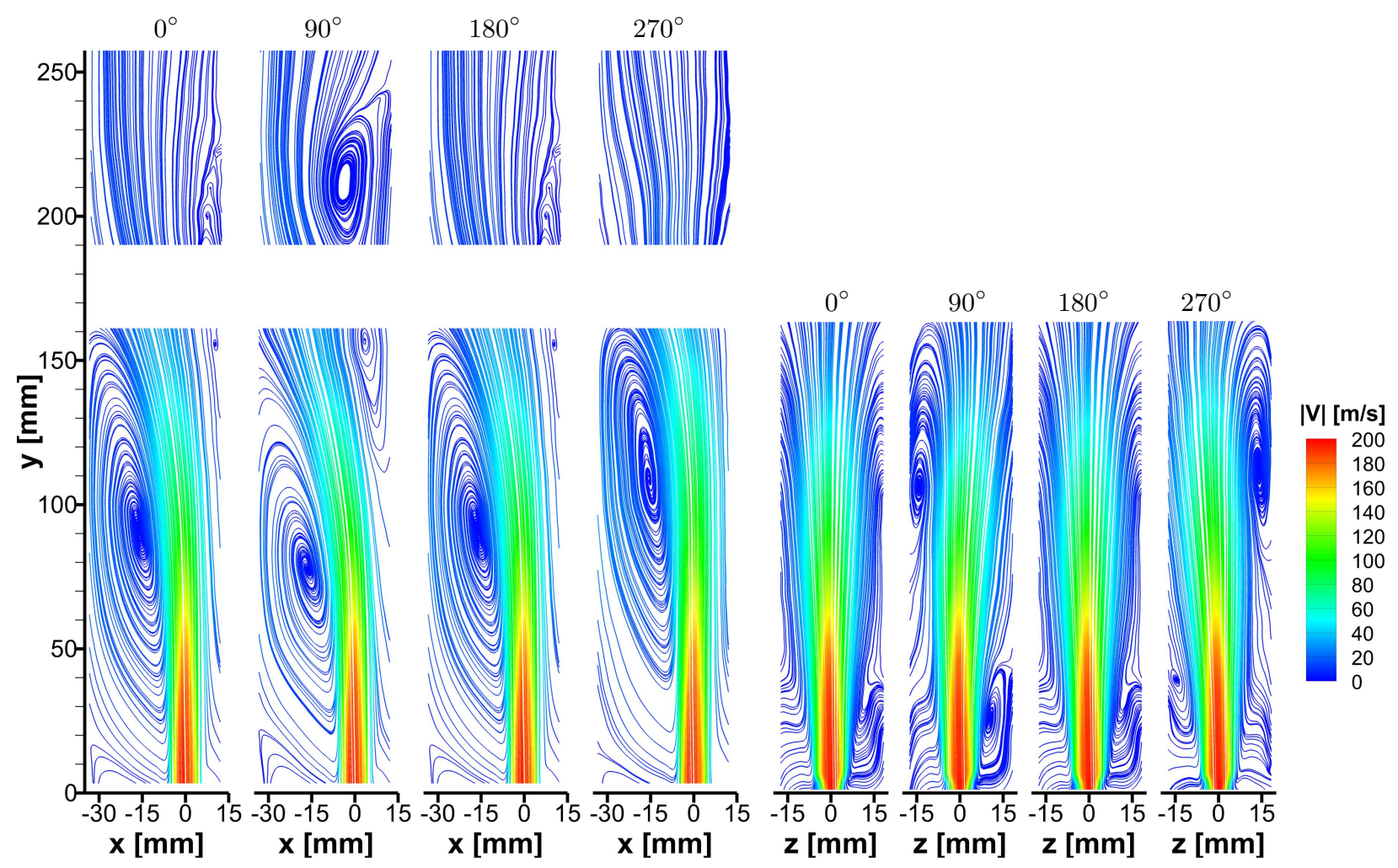

Figure 7: Low-order modeling of phase-dependent flow fields based on the lateral modes

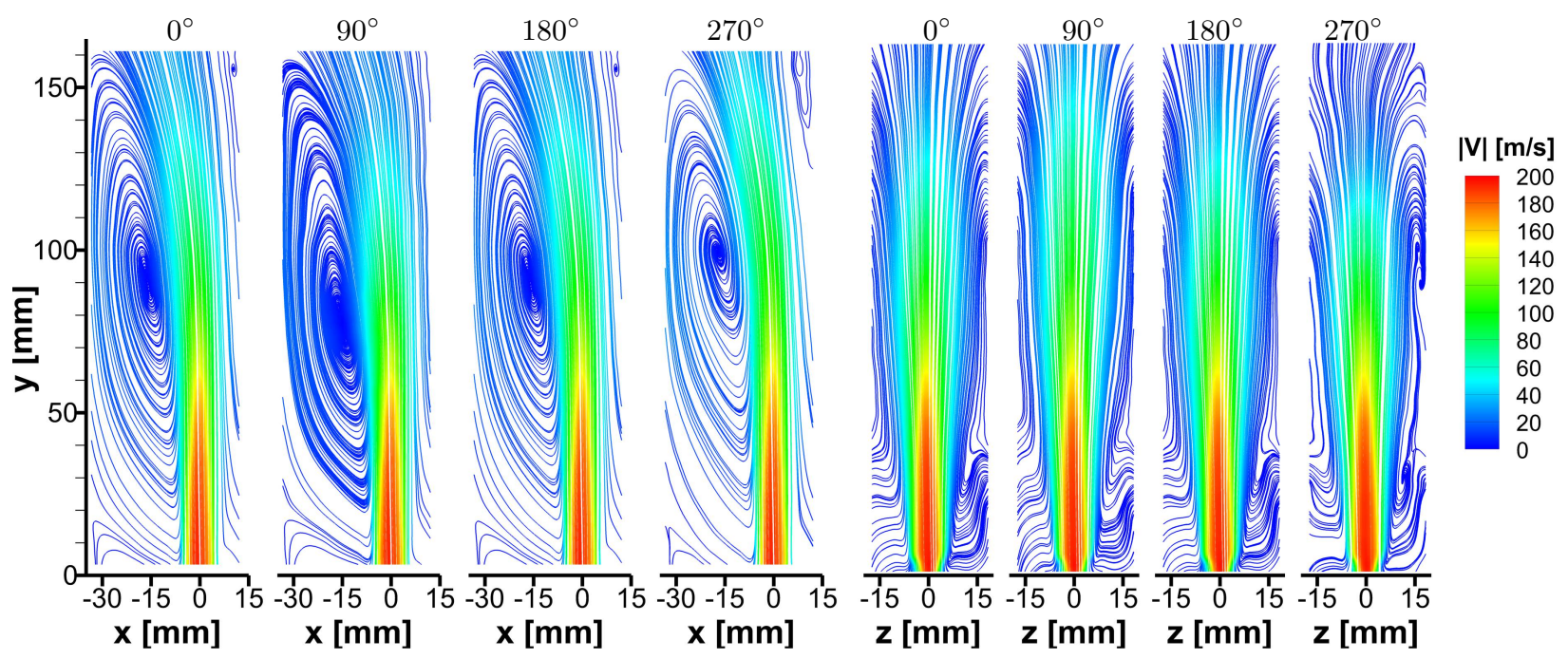

Figure 8: Low-order modeling of phase-dependent flow fields based on the axial modes 
Figure 9: (a) POD spatial modes of the nonreacting flow field in the front view and (b)\&(c) frequency analysis of their temporal coefficients, data taken at $5 \mathrm{kHz}$
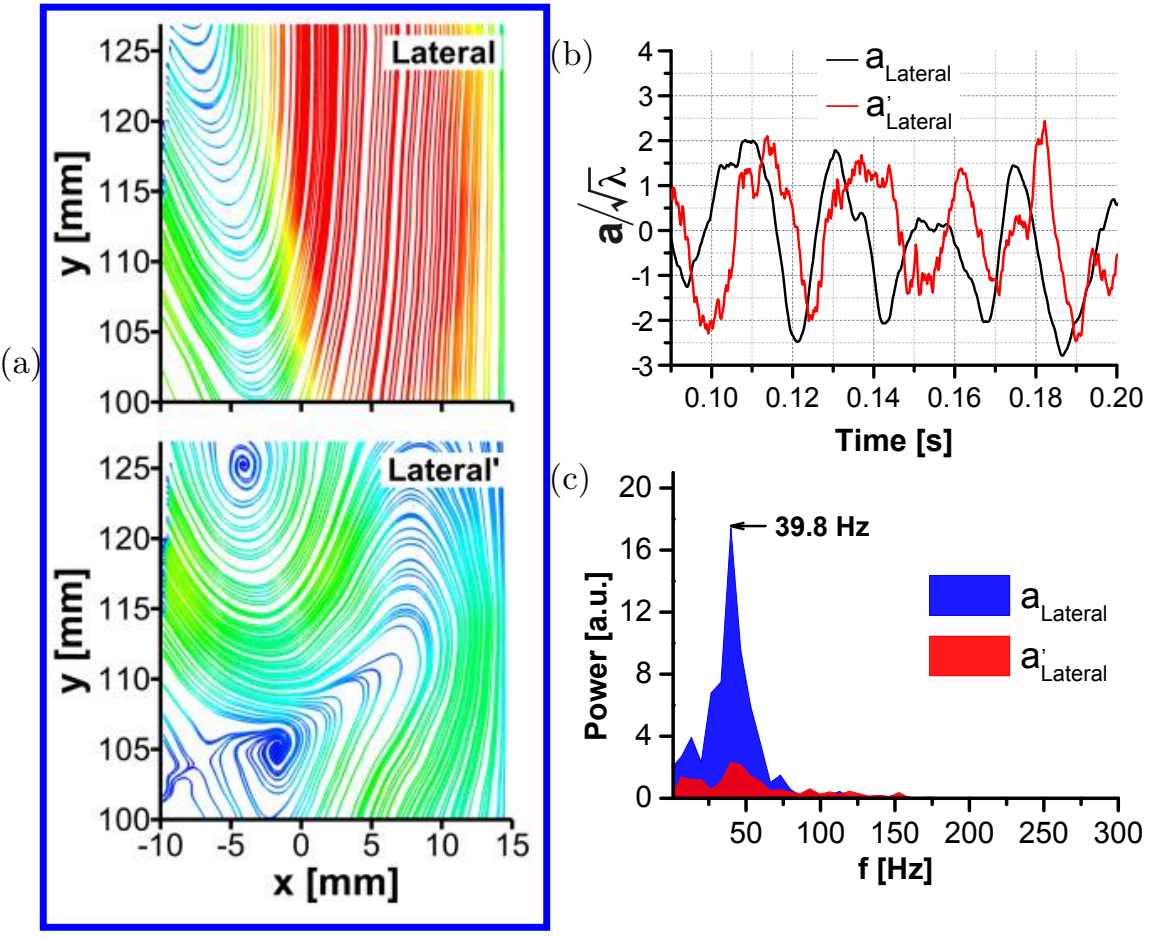

Figure 10: Coupling of the temporal coefficients between the lateral and axial modes of the front view in the non-reacting flow (a)

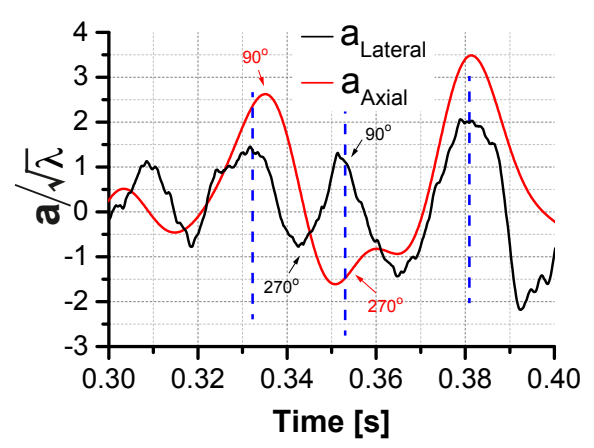

(b)

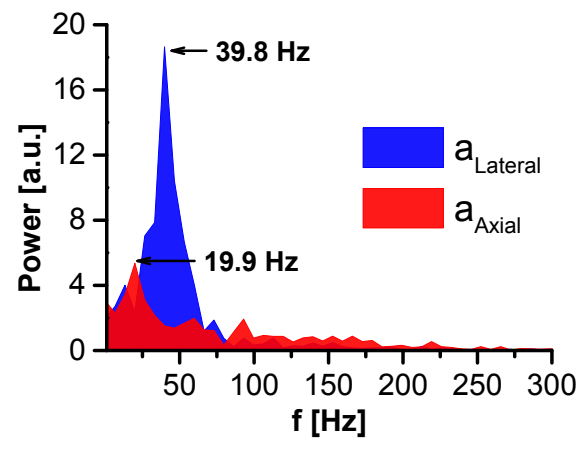

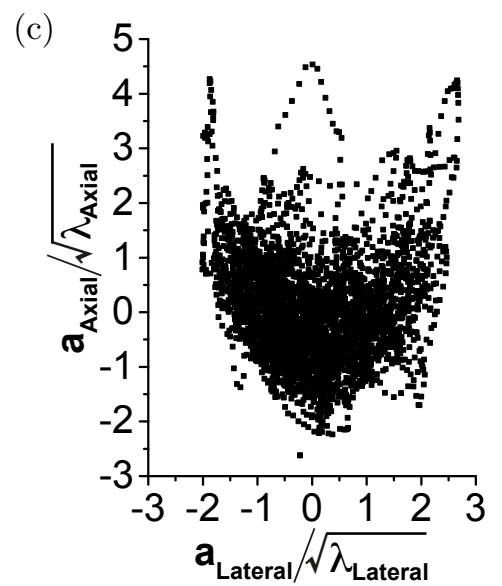

(d)

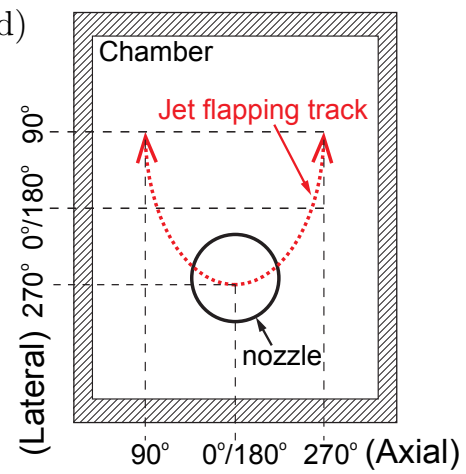


Figure 11: Coupling of the temporal coefficients between the lateral and axial modes of the front view in the reacting flow

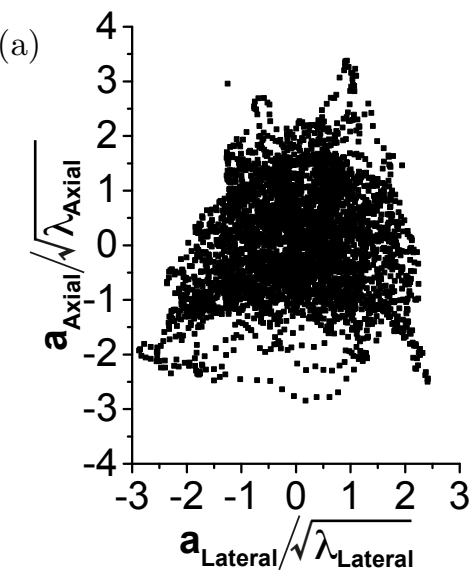

$90^{\circ}$

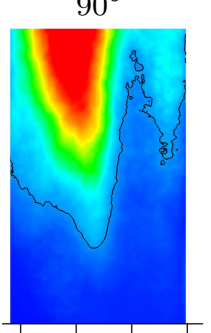

(b)
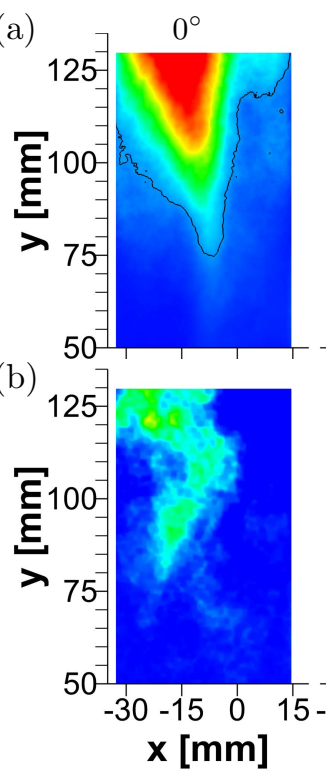

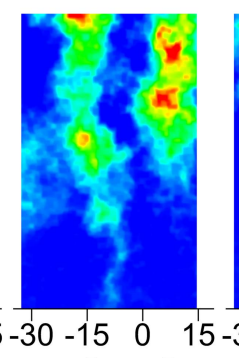

$\mathbf{x}[\mathrm{mm}]$ $180^{\circ}$
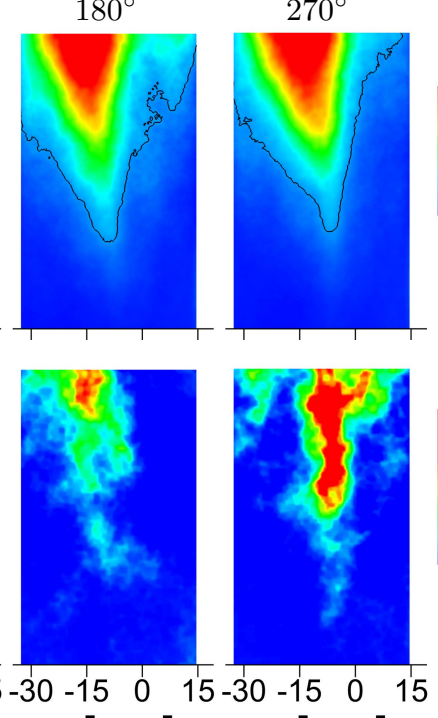

$x$ [mm]

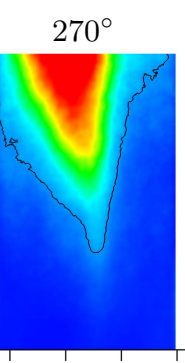

counts

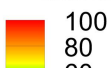

60

40
20

20

counts

counts
300

300
250
200

150

100
50
0 (b)

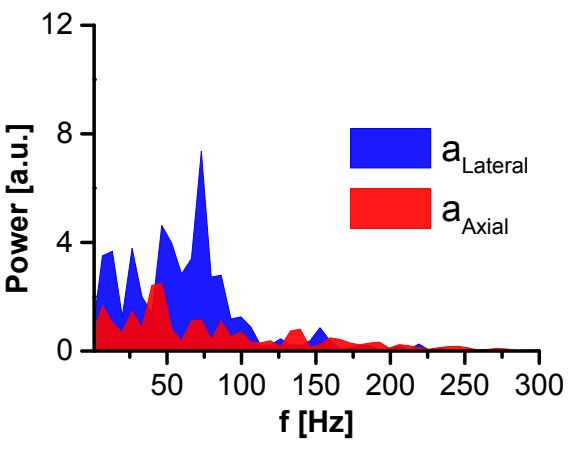

Figure 12: $\mathrm{OH}$ chemiluminescence images at different phase angles: (a) phase averaged based on the temporal coefficients of the lateral mode (b) representative instantaneous shots from different phase groups 
Figure 13: Phase averaged $\mathrm{OH}$ chemiluminescence based on the temporal coefficients of the lateral mode

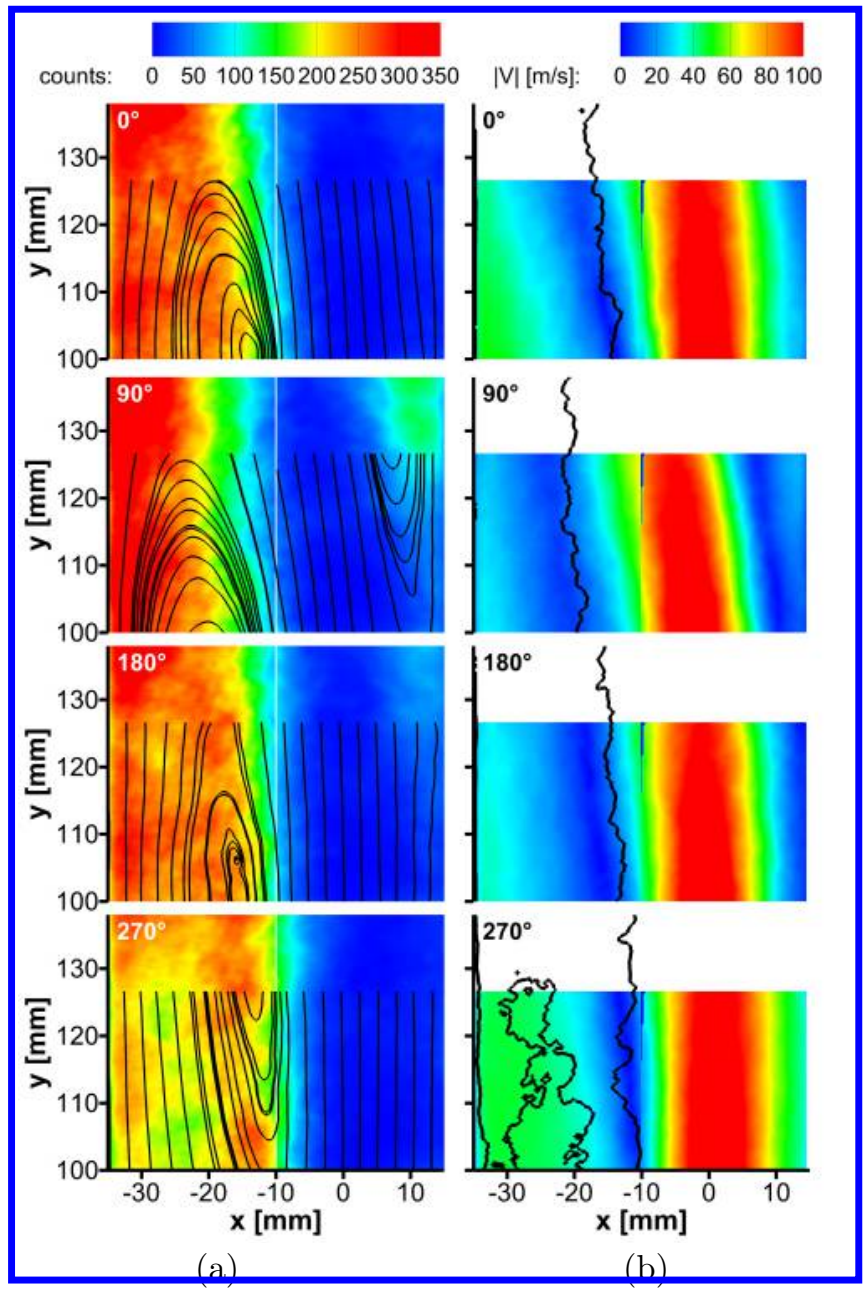




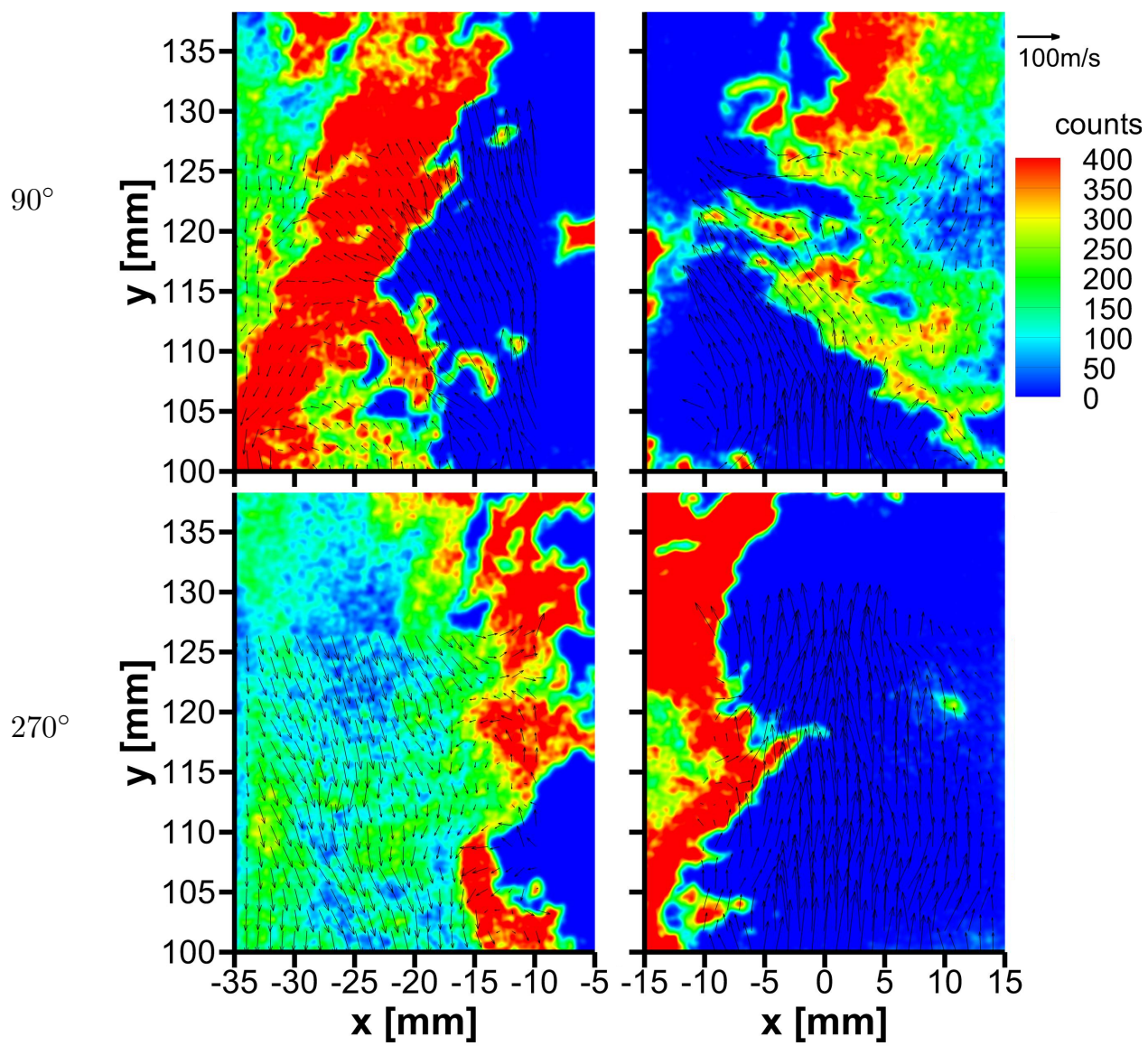

Figure 14: Instantaneous velocity field and $\mathrm{OH}$ distribution chosen $(\mathrm{b})$ from two extreme phase groups.

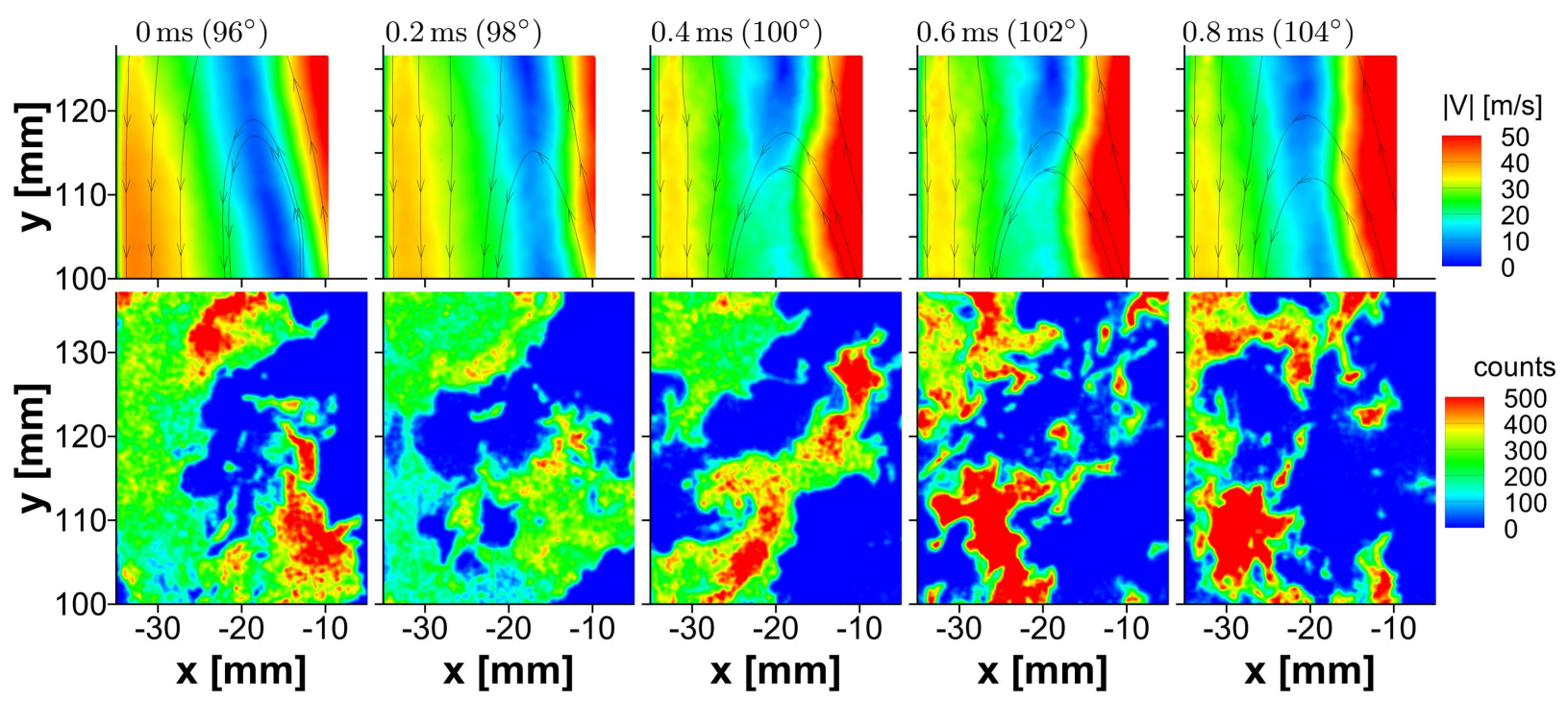

Figure 15: Instantaneous velocity fields and $\mathrm{OH}$ distributions in LRZ depicting flame fragmentation 


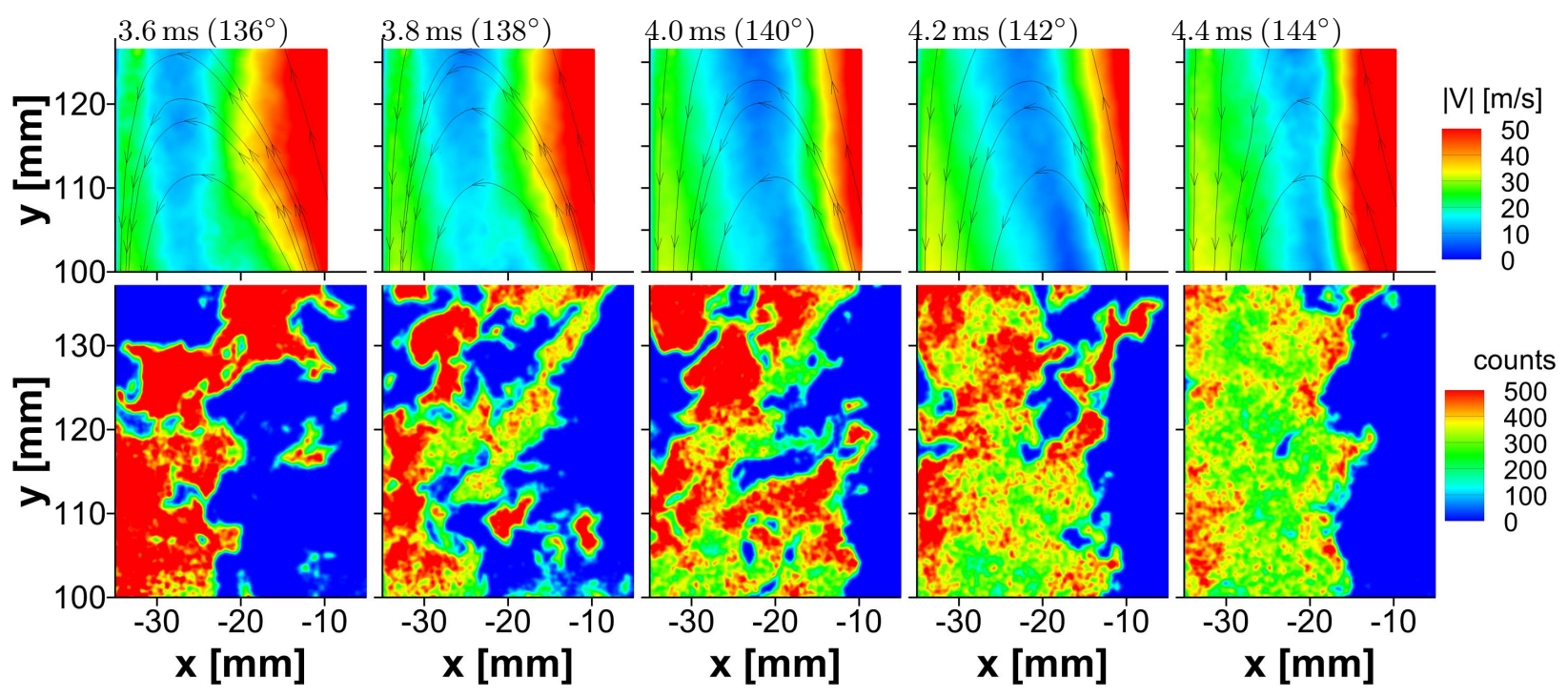

Figure 16: Instantaneous velocity fields and $\mathrm{OH}$ distributions in LRZ depicting flame recovery after temporary local extinction 